\title{
Emprego do questionário CAGE para deteç̧ão de transtornos de USO DE ÁLCOOL EM PRONTO-SOCORRO
}

*G.J. da Paz Filho, L.J. Sato, M.J. Tuleski, S.Y. Takata, C.C.C. Ranzi, S.Y. Saruhashi, B. Spadoni Setordeclínicamédicadopronto-socorrodoHospitalUniversitárioCajuru,Curitiba,PR.

RESUMO - OBjetIvo. Determinar se o questionário CAGE (composto por quatro perguntas de fácil memorização) é capaz de suprir a dificuldade de detecção do alcoolismo.

MATERIAL E MÉTOdo. Realizamos um estudo transversal com amostragem consecutiva em pacientes da Clínica Médica do pronto-socorro do Hospital Universitário Cajuru, os quais foram submetidos a uma entrevista padronizada constituída pelo questionário CAGE e pelo CIDI (Composite International Diagnostic Interview, seções "demografia" e "desordens resultantes do uso de álcool"). Da amostra obtida $(n=374)$, correlacionamos a prevalência de alcoolismo segundo ambos os questionários, considerando o CIDI (critérios do DSM-IV) como sendo o padrão-ouro.
Resultados. A prevalência de dependência de álcool segundo os critérios do DSM-IV foi de $15,77 \%$. Nós verificamos que o CAGE possui sensibilidade igual a $84,74 \%$ e especificidade igual a $73,33 \%$ para um ponto de corte igual a duas respostas afirmativas. Conclusões: Concluímos que o questionário CAGE é um instrumento de fácil aplicação e boa sensibilidade e especificidade quando usado no ambiente de pronto-socorro, podendo suprir as dificuldades de detecção do alcoolismo. Sugerimos que o questionário CAGE seja adotado como rotina no pronto-socorro, permitindo que o índice de detecção de alcoolismo aumente.

Unitermos: Álcool. Alcoolismo. Pronto-Socorro. CAGE. CIDI.

\section{INTRODUÇÃo}

As repercussões dos transtornos decorrentes do uso de álcool (compreendendo abuso e dependência) são bem conhecidas e descritas na literatura, havendo conseqüências de ordens psíquica (como ansiedade, depressão, violência e até suicídio), orgânica (cardiopatias, neuropatias, doença hepática e neoplasias, dentre outras) e socioeconômical-5. Os custos econômicos devidos pelo transtorno são estimados em US\$ 1 66,5 bilhões/ano nos Estados Unidos graças à perda de produtividade (66,9\% do total dispendido), aos gastos em tratamentos e campanhas $(12,7 \%$, sendo os transtornos relacionados ao álcool o terceiro problema mais importante de saúde nos Estados Unidos), aos custos decorrentes de

\footnotetext{
*Correspondência:

R.Ana Berta Roskamp, 1761-Jardim dasAméricas Cep: 81530-250-Curitiba-PR
}

acidentes automobilisticos (9,2\%) e aos crimes relacionados ao consumo de álcool $(8,6 \%)^{4,6,7}$.

Os dados de prevalência do consumo de álcool indicam que, nos Estados Unidos, cerca de 85\% da população geral já consumiu bebida alcoólica pelo menos uma vez na vida 1,2. Dadas as repercussões de ordem social, econômica e médica, vários trabaIhos abordam os diversos aspectos do alcoolismo. A pesquisadora C.J. Cherpitel, da Universidade de Berkeley, tem grande contribuição na área com seus estudos voltados para a análise epidemiológica do consumo de álcool associado à ocorrência de acidentes, principalmente no ambiente de prontosocorro. Além disso, possui trabalhos que pesquisam os diversos métodos de diagnóstico de consumo de álcool (como questionários e dosagem de alcoolemia), buscando o mais efetivo e prático para diversas populações.

A detecção dos transtornos decorren- tes do uso de álcool é um dos primeiros passos a serem dados para que os problemas decorrentes do seu uso sejam minimizados. Entretanto, por se tratar de uma doença cujo diagnóstico depende de uma anamnese detalhada, voltada especialmente para o problema e pela falta de testes complementares eficazes, essa enfermidade é freqüentemente sub-diagnosticada.

Como instrumentos diagnósticos, foram desenvolvidos vários questionários padronizados voltados à detecção dos transtornos decorrentes do uso de álcool, como - CAGE, o MAST, o AUDIT e o TWEAK.

O questionário CAGE ${ }^{8}$ (acrônimo referente às suas quatro perguntas- Cut down, Annoyed by criticism, Guilty e Eye-opener) é utilizado com um ponto de corte de duas respostas afirmativas sugerindo screening positivo para abuso ou dependência de álcool. Segundo a literatura, a sua sensibilidade varia de $43 \%$ a 100\% e a especificidade, de $68 \%$ a $96 \%$, dependendo do tipo de 
amostra estudada9 ${ }^{9-14}$. $O$ trabalho de validação do CAGE no Brasil, realizado por Masur em 1983'5, difere do presente estudo quanto à amostra, pois aquele foi administrado em I I 4 pacientes masculinos de um hospital psiquiátrico, sendo 68 alcoolistas e 46 não-alcoolistas. Além disso, o questionário testado era por constituído por 14 perguntas, com as quatro inquisições do CAGE inseridas nele.

Para determinar se o questionário CAGE possui níveis suficientes de sensibilidade e especificidade, sendo capaz de suprir as dificuldades de detecção de transtornos decorrentes do uso de álcool em prontosocorro, realizamos um estudo transversal em pacientes da clínica médica do prontosocorro do Hospital Universitário Cajuru, submetendo-os a entrevistas constituídas pelo CAGE e pelo $\mathrm{CIDI}^{16}$ (Composite International Diagnostic Interview, referido como o padrão-ouro pela literatura)", analisando em seguida a sensibilidade e a especificidade encontradas.

\section{Materials e Métodos}

Um estudo transversal com amostragem consecutiva foi realizado com pacientes da clínica médica do Hospital Universitário Cajuru, com as mais variadas queixas. De cada quatro pacientes admitidos na clínica médica, um era selecionado e submetido aos critérios de inclusão (pacientes da clínica médica no P.S. que consentiram participar do estudo, maiores de 14 anos) e de exclusão (pacientes não-comunicantes). Tendo satisfeito aos critérios, o paciente era informado da natureza do estudo, e lhe era solicitado consentimento por escrito. Em seguida, cada paciente era submetido a um questionário padronizado composto pelo CAGE (Mayfield et al., 1974) e pelo CIDI (Composite International Diagnostic Interview, Core Version 2. I, seções A-referente a demografia e J-referente a desordens de- vido ao uso de álcool).

O questionário CAGE é constituído por quatro questões referentes ao anagrama Cut-down, annoyed, guilty e eye-opener (Alguma vez o(a) senhor(a) sentiu que deveria diminuir a quantidade de bebida alcoólica ou parar de beber?-cut down; As pessoas o(a) aborrecem porque criticam o seu modo de tomar bebida alcoólica?-annnoyed; $O(a)$ senhor(a) se sente chateado(a) consigo mesmo(a) pela maneira como costuma tomar bebidas alcoólicas?-guilty; Costuma tomar bebidas alcoólicas pela manhã para diminuir o nervosismo ou ressaca?eye-opener). No Brasil, sua validação foi feita em 1983 por Masur e Monteiro ${ }^{15}$, que encontraram uma sensibilidade de $88 \%$ e uma especificidade de $83 \%$. Os pacientes deveriam responder afirmativa ou negativamente às quatro perguntas.

Logo a seguir, era aplicado o CIDI seções $A$ e J, seguindo as recomendações do manual do entrevistador.

As entrevistas foram realizadas durante seis semanas, ficando cada autor responsável pela realização de entrevistas durante um número determinado de turnos, sendo que cada dia era composto por três turnos (manhã: 7h00 às 13h00, tarde: 13 h00 às 19 h00 ou noite 19 h00 às 07 h00 do dia seguinte). Dessa forma, cada um dos 126 turnos das seis semanas teve cobertura por um entrevistador. Esse esquema de amostragem permitiu a obtenção de 374 pacientes.

As respostas obtidas com o CAGE foram analisadas segundo os pontos de corte 1,2,3 e 4, obtendo-se o resultado "CAGE positivo" e "CAGE negativo". Os dados referentes ao CIDI foram digitados no software Ishell 1.0 e o diagnóstico de transtornos decorrentes do uso de álcool foi feito pelo software SPSS 7.5, utilizando os critérios do DSM-IV ${ }^{17}$ para o diagnóstico de dependência de álcool.

Os dados referentes à prevalência de transtornos decorrentes do uso de álcool, segundo o CAGE e o CIDI/DSM-IV, foram cruzados, obtendo-se assim a sensibilidade e especificidade do CAGE.

\section{Resultados}

Dos 374 pacientes da clínica médica do pronto-socorro do Hospital Universitário Cajuru que foram entrevistados, 46,8\% eram homens e 53,2\%, mulheres. A idade média dos pacientes foi de 36,97 \pm 15,63 anos, sendo que a idade mínima encontrada foi de 14 anos e a máxima de 83 anos. Segundo a distribuição por idades, a faixa etária de 14 a 32 anos exclusive engloba a maior porcentagem de pessoas, 44,4\%. Segundo o estado civil, 49,2\% eram casados. Destes, 7,60\% não moravam com o cônjuge. Dos que afirmaram que nunca haviam se casado (32,4\%), 38\% viviam com alguém como se o fosse. 0 número médio de filhos por paciente foi de 2,28 \pm 2,44 , variando de zero a 13 , com a maioria $(45,2 \%)$ dos pacientes tendo I a 3 filhos. A religião mais freqüente foi a católica, com 68, 18\% dos entrevistados. Quanto à escolaridade, a média de anos de estudo foi de 6,63 4 4,43 anos, com média mínima de 0 (zero) anos e máxima de 24 anos, com $62,6 \%$ dos entrevistados tendo estudado de zero a três anos exclusive. Dos 212 $(56,7 \%)$ que referiram não estudar e não ter concluído os estudos, 21 nunca chegaram a estudar. Estavam estudando 5 I entrevistados (13,6\%) e haviam concluído os estudos I I I (29,7\%). A média de idade ao concluir ou interromper os estudos foi de $16,79 \pm 5,58$ anos, variando de 7 a 38 anos, com $51,6 \%$ interrompendo ou concluindo os estudos na idade de 13 a 19 anos exclusive (tabela I).

Referiram estar trabalhando 232 (62,03\%) dos entrevistados. Destes, 12,5\% trabalhavam em meio-período e $87,5 \%$ em período integral. De todos os entrevistados, a média 
do número de meses em que estiveram empregados nos 12 meses anteriores à pesquisa foi de 7,41 \pm 5,23 meses.

Analisando-se as respostas aos questionários CAGE e CIDI, obtivemos uma prevalência de 35,82\% de transtornos decorrentes do uso de álcool para o CAGE (ponto de corte igual a 2) e uma prevalência de 15,77\% para dependência de álcool segundo o CIDI-critérios do DSM-IVI7(figura I).

Analisando-se os diversos pontos de corte, encontramos a seguinte variabilidade: utilizando o ponto de corte I (uma ou mais respostas afirmativas), a sensibilidade encontrada é de $91,52 \%$ e a especificidade, $57,46 \%$. Com o ponto de corte 2, preconizado pela literatura, a sensibilidade encontrada é de $84,74 \%$ e a especificidade, 73,33\%. Já utilizando o ponto de corte 3 , a sensibilidade encontrada é de $57,62 \%$ e a especificidade, $87,61 \%$. Finalmente, com o ponto de corte 4 , a sensibilidade encontrada é de 30,5\% e a especificidade, $97,14 \%$ (figura 2).

Ao se isolar a amostra com mais de 60 anos de idade $(n=39)$, verificou-se uma prevalência de positividade para o CAGE igual a $30,77 \%(n=12)$ e para o CIDI, 10,26\% ( $n=4)$. Nessa amostra, a sensibilidade encontrada foi de $75 \%$ e a especificidade de $74,28 \%$.

\section{Discussão}

O presente estudo tinha como propósito determinar se é vantajoso o uso do questionário CAGE para a detecção de desordens de uso de álcool entre pacientes que procuram o departamento de emergências clínicas de um hospital. Os resultados desta pesquisa indicam que o questionário em questão é útil quando empregado com esse fim. Para avaliar essa afirmação devemos prestar atenção em dois aspectos: eficiência e praticidade na execução.

Assim como em qualquer teste diagnós- tico, a eficiência de um instrumento para a identificação de pacientes portadores de desordens de uso de álcool pode ser analisada pela sensibilidade e especificidade. Com o ponto de corte tradicional, de pelo menos duas respostas afirmativas, foram encontrados valores de $84,7 \%$ para a sensibilidade $\mathrm{e}$ de $73,3 \%$ para a especificidade. Esses achados já sugerem a validade do CAGE na situação descrita neste estudo. Entretanto, ainda é possível obter valores diferentes com o emprego de outros pontos de corte, fazendo com que o questionário se torne mais sensível ou específico, de acordo com finalidade de utilização.

Dentre os diversos pontos de corte, aquele que apresentou melhores especificidade e sensibilidade foi o ponto de corte igual a 2. Os altos valores de sensibilidade $(84,75 \%)$ e de especificidade $(73,33 \%)$ minimizam a inclusão de falsos-positivos e de falsos-negativos na triagem.

Foi realizada a análise separada da amostra constituída pelas pessoas com mais de 60 anos de idade para verificar se o CAGE mantém níveis semelhantes de sensibilidade e de especificidade, haja vista as singularidades encontradas numa amostra

\section{Estado civil}

- casado

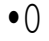

- I a 3

- mais de 3

- $t^{-} 3$ anos

\section{Religião}

- católico

- protestante

- outras

- espírita
Tabela I - Perfil sócio-demográfico da amostra estudada $(n=374)$

\begin{tabular}{|c|c|c|}
\hline Variável & $\mathbf{N}$ & $\%$ \\
\hline \multicolumn{3}{|l|}{ Sexo } \\
\hline - masculino & 175 & 46,8 \\
\hline •femino & 199 & 53,2 \\
\hline \multicolumn{3}{|l|}{ Idade } \\
\hline$\cdot 14^{\vdash} 32$ anos & 166 & 44 \\
\hline - $32^{\vdash} 50$ anos & 128 & 34 \\
\hline - $50^{\vdash} 68$ anos & 57 & 15 \\
\hline - $68 \vdash 86$ anos & 23 & 6,2 \\
\hline
\end{tabular}

- nunca se caso

121

32,4

- separado/divorciado

184

45

6,4

\section{Número de filhos}

111

29,7

169

45,2

94

25,1

234

62,6

$\cdot 3-6$ anos

$100 \quad 26,7$

- $6^{-} 9$ anos

7,8

29

2,9

255

68,2

75

20

2,4

9

0,8 
cation Test, com 10 perguntas) e o MAST (Michigan Alcohol Screening Test, com 25 perguntas), apresentando desempenho bem próximo ao descrito para esses instrumentos ${ }^{18-20}$.

Apesar da prevalência de alcoolismo ser alta, não só entre a população geral, mas também entre a população atendida em pronto-socorro, essa condição é raramente pesquisada em tal ambiente. A sua detecção em pacientes de pronto-socorro é de grande importância no atendimento médico, pois constitui em ótima oportunidade de diagnóstico precoce desses distúrbios, permitindo uma possível intervenção médica contra o problema, ou até mesmo o encaminhamento do paciente a um serviço especializado. Tendo em vista que o alcoolismo é fator de risco para diversas enfermidades, a sua detecção não só auxiliaria no diagnóstico e tratamento de suas conseqüências no próprio ambiente de pronto-socorro, mas também possibilitaria a prevenção dessas enfermidades através da intervenção precoce, seja no pronto-socorro, seja em um serviço especializado. Estudando instrumentos para avaliação de transtornos decorrentes do uso do álcool, trabalhos mostram que questionários padronizados (como o CAGE) são mais sensíveis e específicos do que a determinação dos níveis de alcoolemia ${ }^{21}$, além de serem práticos, baratos e acessíveis a todos os serviços de emergência. Outros estudos, como o realizado no Hospital Geral de San Francisco, Califórnia, mostram que a pesquisa da alcoolemia pelo ar exalado, associada ao relato voluntário de consumo de álcool, pode ser apropriada na detecção de consumo de álcool recente ${ }^{22}$.

Em relação ao desenho e execução do estudo devem ser levados em consideração alguns fatos que podem ter causado certa distorção dos achados. Um deles foi a seqüência de aplicação dos questionários (primeiro o CAGE e em seguida o CIDI), fazen-

Gráfico I - Prevalência de positividade para o CAGE (ponto de corte igual a 2) e para o CIDI.

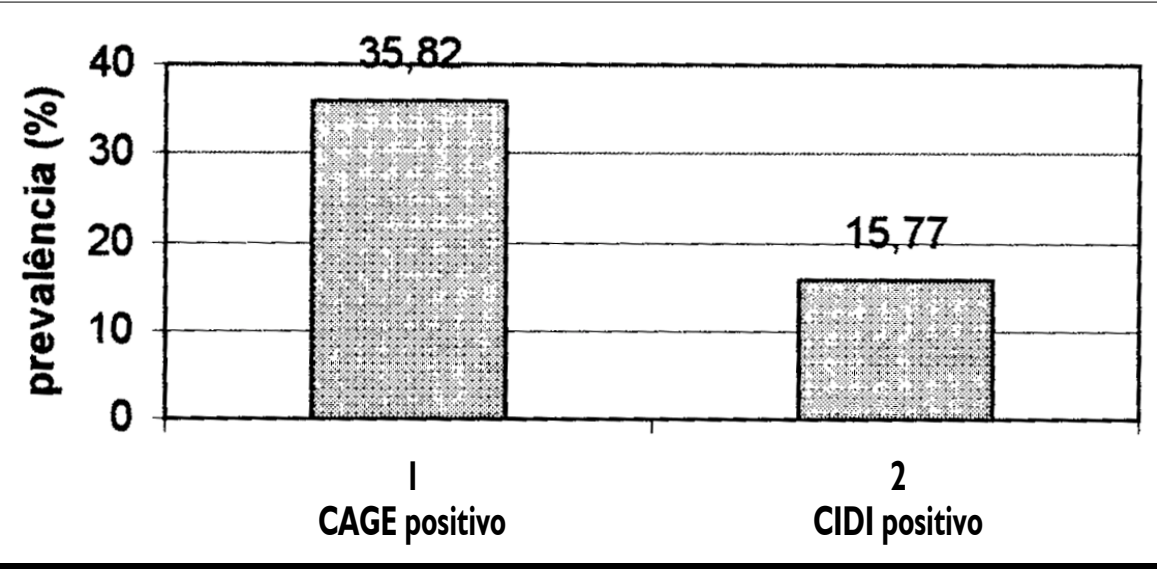

Gráfico 2 - Sensibilidade e especificidades do CAGE de acordo com os pontos de corte.

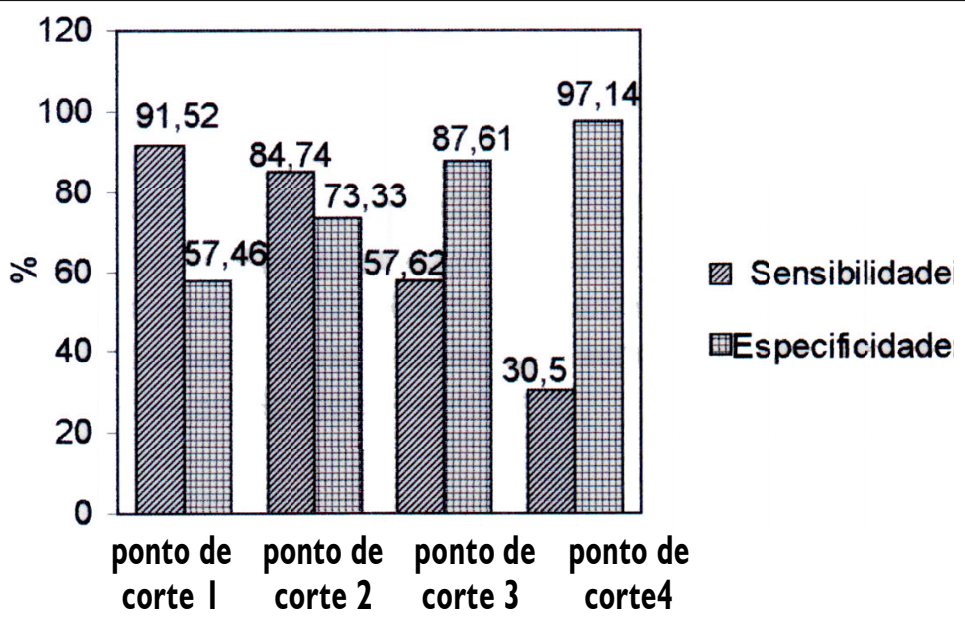

do com que os indivíduos, muitas vezes se sentissem mais à vontade com o decorrer das perguntas, dando informações de confiabilidade diferente nos dois tempos da entrevista. Além disso, existiam pacientes que se afligiam com a extensão do CIDI e passavam a fornecer respostas negativas com o intuito de abreviar a pesquisa. Outra falha foi a não-tabulação dos dados referentes aos pacientes que foram selecionados pela amostragem consecutiva, mas que não foram entrevistados, bem como a nãolistagem os motivos. A inexistência de trabaIhos de validação do CIDI para o língua portuguesa também constitui em obstáculo para a realização do trabalho, obrigando os autores a traduzir os referidos questionários já validados nas línguas inglesa e espanhola. Entretanto, verificamos que dentre esses motivos, o principal foi a impossibilidade de comunicação, seguida por transferência ou alta antes da entrevista e recusa.

\section{Conclusóes}

Nós encontramos resultados sugestivos de que o questionário CAGE pode ser útil para identificação de pacientes com desordens de uso de álcool no departamento de emergências clínicas. Levando em consideração que tanto a anamnese convencional 
quanto os sistemas de aferição dos níveis sangüíneos de álcool podem não ser confiáveis para o diagnóstico desse tipo de desordem?, além de outros questionários serem extensos e de difícil aplicação, as quatro indagações do CAGE têm aplicabilidade factível, associada a bons resultados práticos aparentemente.

\section{SUMMARY}

\section{USE OF THE CAGE QUESTIONNAIRE FOR DETECTING ALCOHOL USE DISORDERS AT THE EMERGENCY ROOM.}

BACKGROUND. Alcoholism is seldom detected among emergency room outpatients, which is attributed to the non-adoption of diagno stic routines, to the difficulty of doing a special anamnesis for diagnosing alcoholism, and to the lack of sensitivity and specificity of the laboratory tests. This study has the purpose to determine whether the CAGE assay (composed by 4 questions of easy memorization) is able to supply this difficulty in detecting alcoholism.

Material and methods. We did atransversal study with consecutive sampling among outpatients of the Internal Medicine Department at Cajuru University Hospital emergency room. They were submitted to a standardized interview constituted by the CAGE and the CIDI assays (Composite International Diagnostic Interview, "demographics" and "disorders resulting from the use of alcohol" sections). From the sample obtained ( $n=374)$, we correlated the prevalence of alcoholism according to both questionnaires, considering the CIDI (DSM-IV criteria) to be the gold standard.
REsULTS. The prevalence of alcohol dependence according to the DSM-IV criteria was $15.77 \%$. We verified that the CAGE has $84.74 \%$ of sensitivity and $73.33 \%$ of specificity, with a cut point of 2 positive responses.

Conclusions. We concluded that the CAGE is an instrument of easy application and good sensitivity and specificity when used at the emergency room, being able to supply the difficulties of alcoholism detection. We suggest that the CAGE be adapted as a routine at emergency rooms, increasing the alcoholism detection rate.

KEY wORDS: Alcohol. Alcoholism. Emergency department. CAGE. CIDI.

\section{ReferênCias Bibliográficas}

I. Kaplan, H.I. Transtornos relacionados a substâncias. In Kaplan, H.I., Sadock, B.J., Grebb, J.A. eds. Compêndio de Psiquiatria-Ciências do comportamento e Psiquiatria Clínica. 7ª ed. Porto Alegre, Artes Médicas, 1997; 381-95.

2. National Institute of Alcohol Abuse and Alcoholism. News release. 1998.

3. Morse, RM, Flavin, DK. The definition of alcoholism. JAMA, 1992; 8(268): I012-4.

4. McGinnis, J, Foege, W. Actual causes of death in the United States. JAMA, 1993; 1 8(270): 2208.

5. Wallerstedt, S, Denison, H, Sandstrom, J, Wsetin, J. The prevalence of alcoholism and its relation to cause of hospitalization and longterm mortality in male somatic inpatients. J Intern Med, 1995; 237: 339-44.

6. U.S. Department of Justice. Alcohol and crime: an analysis of national data on the prevalence of alcohol involvement in crime. 1998

7. National Institute of Alcohol Abuse and Alcoholism. Eighth special report.

8. Mayfield, D, McLeod, G, Hall, P. The CAGE questionnaire: validation of new alcoholism screning instrument. Am J Psychiatry, 1974; |3|: ||2|-3.

9. Schorling, JB. Screening for alcohol and drug abuse. Medical Clinics of North America, 1997; 4(81): 845-65.

10. Liz, MA, Coutinho, ESF. Prevalência de consumo de bebidas alcoólicas e de alcoolismo em uma região metropolitana no Brasil. Rev. Saúde Pública, 1993; 27:23-9.

II. Cherpitel, C.J. Screening for alcohol problems in the emergency department. Ann Emerg Med, 1995; 26(2): 158-66.

12. Buschsbaum, DG, Buchanan, RG, Centor, RM et al. Screening for alcohol abuse using CAGE scores and likelihood ratios. Ann Intern Med, 199|; I0(|| 5): 774-7.

13. Wallace, $P$, Haines, A. Use of a questionnaire in general practice to increase the recognition of patients with excessive alcohol consumption Brit Med J, 1985; 29(290): 1949-52.

14. Liskow, B, Campbell J, Nickel EJet al. Validity of the CAGE questionnaire in screening for alcohol dependence in a walk-in (triage) clinic. J Stud Alcohol, 1995; 56: 277-8I.

15. Masur, J, Monteiro, M. Validation of the CAGE alcoholism screening test in Brazilian Psychiatry inpatient hospital setting. J Biol Res, 1983; 16: 215-8.

16. Composite International Diagnostic Interview (CIDI). Geneva: World Health Organization, 1989

17. Transtornos Relacionados a substâncias. In Manual de diagnóstico e estatística de doença mental, 4a ed. Portos Alegre, Artes Médicas 1995; 171-263.

18. Morton, JL, Jones, TV, Manganaro, MA. Performance of alcoholism screening questionnaires in elderly veterans. Am J Med, 1996; 101: 153-159.

19. Schmidt, A, Barry, KL, Fleming, MF. Detection of problem drinkers: the alcohol use disorders identification test (AUDIT). South MedJ, I995; 88:52-59.

20. Bohn, M. J. The alcohol use disorders identification test (AUDIT): Validation of a screening instrument for use in medical settings. J Stud Alcohol, 1995; 56: 423-32.

21. Cherpitel CJ, Soghikian K, Hurley LB. Alcoholrelated health services use and identification of patients in the emergency department. Ann Emerg Med, 1996; 28(4): 418-23.

22. Cherpitel C). Breath analysis and self-reports as measures of alcohol-related emergency room admissions. J Stud Alcohol , | 989; 50(2): | 55-6 |

Artigo recebido: 09/09/1999

Aceito para publicação: 17/10/2000 